\title{
Segmentation of Brain MRI in Young Children
}

\author{
Maria Murgasova ${ }^{1}$, Leigh Dyet ${ }^{2}$, David Edwards ${ }^{2}$, Mary Rutherford ${ }^{2}$, \\ Joseph V. Hajnal ${ }^{2}$, and Daniel Rueckert ${ }^{1}$ \\ ${ }^{1}$ Visual Information Processing Group, Department of Computing, \\ Imperial College London \\ ${ }^{2}$ Department of Imaging Sciences, Faculty of Medicine, Imperial College London
}

\begin{abstract}
This paper describes an automatic tissue segmentation algorithm for brain MRI of young children. Existing segmentation methods developed for the adult brain do not take into account the specific tissue properties present in the brain MRI of young children. We examine the suitability of state-of-the-art methods developed for the adult brain when applied to the segmentation of the young child brain MRI. We develop a method of creation of a population-specific atlas from young children using a single manual segmentation. The method is based on non-linear propagation of the segmentation into population and subsequent affine alignment into a reference space and averaging. Using this approach we significantly improve the performance of the popular EM segmentation algorithm on brain MRI of young children.
\end{abstract}

\section{Introduction}

The problem of automatic segmentation of brain MRI has been extensively studied in past decade thanks to its applications in clinical studies of normal and diseased brain. Recent research of brain development in prematurely born children requires reliable and accurate automatic segmentation techniques for the neonatal and early childhood brain as well. Approximately $40 \%$ of prematurely born children have cognitive, neurological or behavioural impairment [1] and the cerebral abnormalities underlying problems such as minor motor impairment or inattention still remain unclear. Accurate tissue quantification of the preterm brain in early childhood would allow us to study the growth of different structures and help to relate developmental outcomes to changes in volume and shape of anatomical structures.

In addition to noise, intensity non-uniformity, partial volume effect and natural tissue intensity variation present in the adult brain MRI, neonatal and infant brain MRI tissue intensities have even greater natural variation caused by ongoing process of myelination of white matter. This is most profound in neonates where white matter is extremely difficult to distinguish from grey matter on T1 weighted MRI sequences. By the age of 1 or 2 years, the majority of white matter has myelinated. However, the natural tissue intensity distribution overlap results in large misclassifications in central brain structures. This problem is further compounded by the fact that child brain differs significantly in size and 
shape from the adult brain, in particular in the central structures of the brain [2]. This causes problems for methods which rely on probabilistic atlases built from adults as spatial prior information.

In this paper we focus on the segmentation of brain MRI of 1 and 2-yearold children. We show how to bootstrap a probabilistic tissue atlas for a specific population based on registration of a single manual segmentation to a population and subsequent alignment to the space of a reference subject and averaging. We demonstrate that the results of the expectation-maximization method by Van Leemput et al. (3, 4]) on young children can be significantly improved by using an atlas which appropriately captures the anatomical variability of the infant brain and compare the results with a simple version of a method inspired by Tasdizen et al. [5].

\section{Related Work}

To date the research in MRI brain segmentation has focused on adult and neonatal brains. The most popular class of tissue classification methods is based on expectation-maximization framework $([3$, , 4, 6], 7]) which iteratively interleaves calculation of intensity probability distributions and/or bias field with refinement of spatial probability maps. A mixture Gaussian distribution is assumed as a model for the voxel intensity probability distribution as it is easy to incorporate into EM framework. However, the real distribution can significantly differ from Gaussian for example due to partial volume effect, see Fig. 1. This can be overcome by estimating the real distributions [7] or by including a model for the partial volume effect in the EM framework [8].

Natural tissue intensity variation in different areas of the brain is another reason for misclassification. For example, grey matter in central brain structures is characterized by brighter intensities than cortical grey matter, and is therefore likely to be misclassified as white matter. Ren et al 9] reports such misclassification in adult brain using the EM-based segmentation method FAST [10]. However non-rigid registration-based label propagation [1] succeeds in this area of the brain as it does not assume any model for tissue intensities.

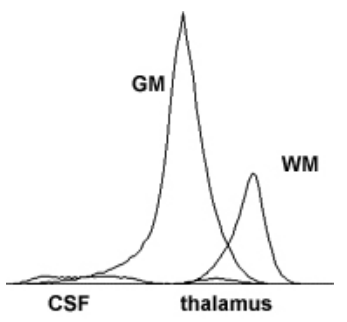

Fig. 1. Histogram of the tissue intensity probability distribution for white matter, grey matter, cerebrospinal fluid and thalamus (a deep grey matter structure) based on a manual segmentation of brain MRI from a 2-year-old child. Distributions of grey matter and CSF differ significantly from the Gaussian distribution due to partial volume effect. The intensity distribution of thalamus lies in the region of the overlap of the white and grey matter distributions.

Inevitable misclassifications resulting from overlaps in tissue intensity distributions can be partially avoided by spatially constraining the segmentation process with prior information in form of an aligned probabilistic atlas at each 
iteration ([3], 4]); however this makes the method very sensitive to the correct alignment of the atlas with the image. This is very difficult for child brains which significantly differ from adult brains in size and shape 2. To solve this problem, 12 and 13 suggest to warp the atlas to subject by non-rigid registration. In 14 Pohl reports difficulties with non-rigidly registering the template to image which is consistent with our own experience. Recent methods try to overcome this problem by iteratively warping a deformable atlas and refining the segmentation at the same time [15, [16].

An alternative non-parametric method for adult brain classification was developed by Cocosco et al. [17. In this method the atlas is used only to select the training samples which are consequently used in $\mathrm{kNN}$ classification. This method is reported to deal well with anatomies which differ from the prior information. However, it cannot deal with the problem of tissue intensity distribution overlap in single-channel T1 data. Similarly, Tasdizen et al. [5] use the atlas only to initialize the estimation of multi-dimensional distribution of 7-neighbourhood.

The adult brain segmentation methods can be applied to the neonatal and infant brain when tuned to their specific properties. This was shown by Prastawa et al. [18] who used the EM approach ([3], 4]) with an atlas derived from single subject segmentation used as a prior, followed by non-parametric classification similar to [17 on neonatal data.

\section{Methods}

\subsection{Expectation-Maximization Algorithm}

For segmenting T1-weighted images from 1- and 2- year old children we have chosen the EM algorithm by Van Leemput et al.(3], [4]). We aligned the CCHMC pediatric brain atlas created from population of 5 to 9 -year-old children [2] (Fig. 4.) with each subject's image. All images acquired on 1.0T HP MRI scanner were intensity corrected by the N3 software [19] in preprocessing step which proved to be sufficient as the bias field was not very strong. We have also acquired images on a $0.5 \mathrm{~T}$ Apollo MRI scanner. In this case no bias field correction was necessary. This allowed us to study the EM method without the bias correction step and consequently better analyze its effect on the resulting segmentation.

The EM algorithm is a general technique for finding missing data based on observed data and maximum likelihood parameters estimates. In our case the observed data are the image intensities, the missing data are the labels and the parameters are the means and variances of the Gaussian distribution which is assumed for the intensity distribution of each tissue class. This is an iterative process which interleaves the calculation of posterior probabilities of each voxel belonging to each tissue class (white matter, grey matter, cerebrospinal fluid, other) - the expectation step, with maximum likelihood estimation of the Gaussian distribution parameters - the maximization step. 
E-step:

$$
p_{i k}^{m}=\frac{G\left(y_{i}, \mu_{k}^{m-1}, \sigma_{k}^{m-1}\right) p_{i k}^{a t l a s}}{\sum_{j} G\left(y_{i}, \mu_{j}^{m-1}, \sigma_{j}^{m-1}\right) p_{i j}^{a t l a s}}
$$

M-step:

$$
\mu_{k}^{m}=\frac{\sum_{i} p_{i k}^{m} y_{i}}{\sum_{i} p_{i k}^{m}} \quad \sigma_{k}^{m}=\frac{\sum_{i} p_{i k}^{m}\left(y_{i}-\mu_{k}^{m}\right)^{2}}{\sum_{i} p_{i k}^{m}}
$$

where $p_{i k}^{m}$ denotes the posterior probability of $i^{\text {th }}$ voxel belonging to tissue $k$ at $m^{\text {th }}$ iteration, $y_{i}$ is an intensity of $i^{\text {th }}$ voxel and $G(., .,$.$) denotes the Gaussian$ distribution with the mean $\mu_{k}^{m}$ and variance $\sigma_{k}^{m}$.

We found that this method significantly overestimates white matter in the central structures of the brain. This is most profound in the thalamus, a deep grey matter structure, where about $30 \%$ of voxels were misclassified as white matter (see Fig. 2 and Fig.4b). The histogram based on the manual segmentation of a 2year-old 1.0T data set (Fig. 1) shows that the voxel intensities of this structure lie in the region of the overlap of white and grey matter intensity distributions and therefore correct classification in this area can be achieved only by using correct prior information. However, prior information from the probabilistic atlas [2] overestimates white matter in this area as it does not describe the anatomy of the brain in early childhood adequately (Fig. 4k).

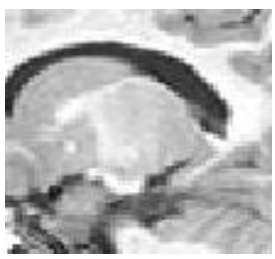

(a)

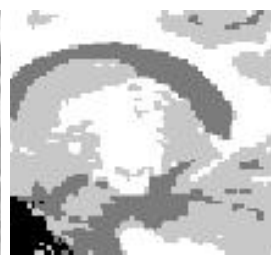

(b)

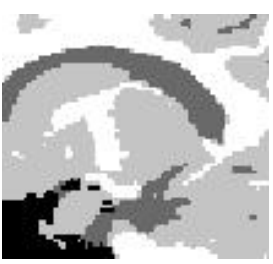

(c)

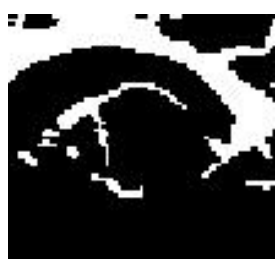

(d)

Fig. 2. Misclassification in thalamus: Substantial amount of deep grey matter classified as white. (a) MR image of a 2-year-old brain (b) EM segmentation using the adult atlas (c) EM segmentation using the 2-year-old child atlas (d) manual segmentation.

\subsection{Bootstrapping the Prior Information for 1 and 2-Year-Old Brain}

We obtained a manual segmentation of a single 2-year-old subject to create a probabilistic atlas of the 1 and 2-year-old brain. The manual segmentation was then transformed to 37 2-year-old subjects by performing non-rigid registration [11. All 37 segmented subjects were then aligned with the reference subject by affine registration and the segmentations were averaged. The resulting probability maps describe the anatomy of the 2-year-old brain correctly when aligned with a subject by affine registration.

We also created a separate probabilistic atlas of the 1-year-old brain. First we non-rigidly warped the manual segmentation of a 2 -year-old brain to the scan of 
the same subject at 1 year to minimize the registration error. The same process of the atlas creation was then repeated with 341 -year-old scans.
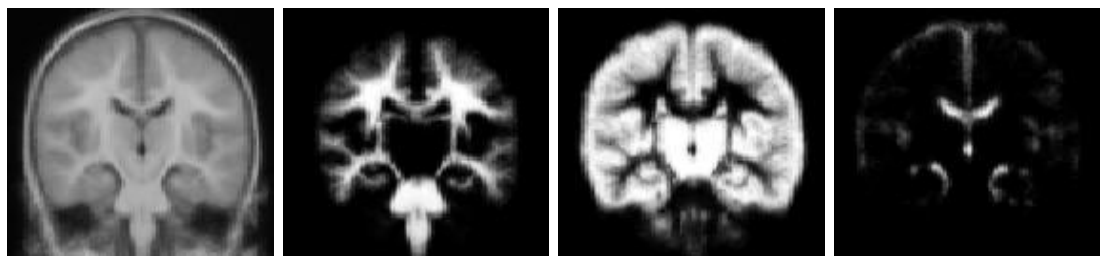

Fig. 3. The probabilistic atlas created from population of 2-year-old children

We were also able to use the single subject segmentation as the prior information by registering it non-rigidly to the image and subsequent blurring to accommodate anatomical variability.

Both of these methods resulted in significant improvement in segmentation of the central brain structures (Fig. 2 and 4). However, the white matter was still consistently overestimated which is caused by trying to separate the tissue classes based on single voxel intensity on global scale.

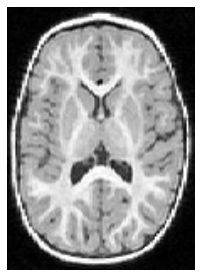

(a)

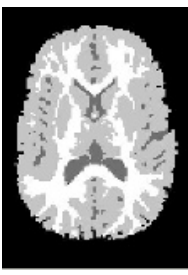

(b)

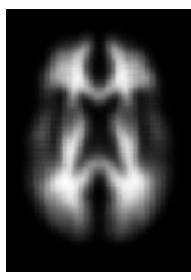

(c)

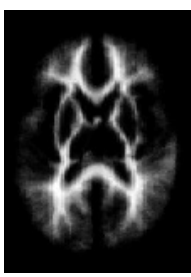

(d)

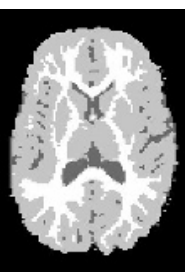

(e)

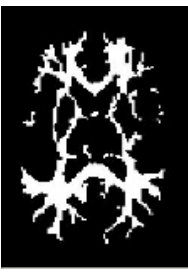

(f)

Fig. 4. Improvement of segmentation using our new atlas: (a) MR image of a 2-yearold brain (b) segmentation using the 5 to 9 -year-old CCHMC brain atlas (c) the 5 to 9-year-old CCHMC brain atlas (d) our 2-year-old brain atlas (e) segmentation using our 2-year-old brain atlas (f) manual segmentation

\subsection{Local Distribution Estimation Method}

In early childhood brain MRI exhibits significant natural intensity variability within the tissue classes. However, the boundaries are often visible to human observer. Therefore local estimation of tissue distributions can improve the segmentation. Inspired by [5] we implemented a local distribution estimation method which we have compared with the results of the EM approach in previous sections. A non-rigidly warped manual segmentation was used as prior information and the tissue intensity distributions were then estimated by Parzen windowing for each voxel separately after masking with local Gaussian kernel. Each voxel was then assigned to the tissue class with the highest probability. Even though 
this approach improved the segmentation in small detail it was also less stable and sensitive to noise than the methods which estimate the tissue intensity distributions globally.

\section{Validation and Results}

\subsection{Validation Using Manual Segmentation}

First, we have validated our results on a single subject for which we obtained the manual segmentation. Validation was performed only in the brain area, excluding skull, brain stem and cerebellum. We segmented the image with our implementation of the EM method (section 3.1) using 3 different atlases as prior: our new probabilistic atlas based on population of 2-year-old children (2yr atlas), blurred manual segmentation (2yr seg) and the standard adult atlas obtained from SPM software 20] (adult atlas). For comparison we also segmented the image with EMS software [21] developed by Van Leemput. This software uses the SPM adult atlas, and unlike in our implementation, the bias correction is incorporated within the EM framework. We validated the approach with and without Markov Random Fields (MRF). Segmentation using local estimation of tissue parameters was evaluated as well. The manual segmentation was used as the ground truth. The correctness of the segmentation was calculated using Dice metric

$$
D=\frac{2 *\left|T_{g t} \cap T_{\text {seg }}\right|}{\left|T_{g t}\right|+\left|T_{\text {seg }}\right|}
$$

where $T_{g t}$ and $T_{\text {seg }}$ denote the set of samples of given tissue type in the ground truth and the automatic segmentation, respectively. The validation results (Fig. 19) show that our new atlas improved the segmentation of both white and grey matter by $4 \%$ compared to EM segmentation with the adult atlas by both EMS and our implementation. The improvement is most significant in the thalamus where performance increased by $12 \%$ in comparison with EMS and by $26 \%$ compared to our implementation. Better performance of the EMS software in comparison with our implementation of EM with the adult atlas can be explained by the fact that the bias correction step partially compensates for natural intensity variation in the infant brain. When a blurred manual segmentation was used as prior information for EM, the performance slightly increased, but in this case it did not have to be non-linearly warped to the subject and therefore the results do not include the registration error. Overall performance of the local method was only marginally better than EMS due to sensitivity to noise and instability. However, it resulted in $94 \%$ correct segmentation of the thalamus. Again, the results do not include the registration error.

\subsection{Two Step Validation}

In the second experiment we compared our 3 methods on 37 2-year-old subjects. As we do not have the ground truth for any other subject we performed a two step segmentation. First, we segmented all the subjects by each of our methods and 
Table 1. (a) Performance of the segmentation methods on a single 2-year-old subject (b) Two-step validation of the segmentation methods on 37 2-year-old subjects

\begin{tabular}{l|c|c|c|c} 
& WM & GM & CSF & thalamus \\
\hline EMS & 0.844 & 0.883 & 0.694 & 0.779 \\
EMS-MRF & 0.855 & 0.891 & 0.616 & 0.799 \\
EM adult atlas & 0.847 & 0.879 & 0.672 & 0.664 \\
EM 2yr atlas & 0.885 & 0.922 & 0.784 & 0.923 \\
EM 2yr seg & 0.904 & 0.927 & 0.814 & 0.926 \\
local & 0.877 & 0.906 & 0.625 & 0.939
\end{tabular}

(a)

\begin{tabular}{l|c|c|c|c} 
& WM & GM & CSF & thalamus \\
\hline EM 2yr atlas & 0.716 & 0.852 & 0.569 & 0.878 \\
EM 2yr seg & 0.872 & 0.907 & 0.740 & 0.847 \\
local & 0.855 & 0.809 & 0.404 & 0.916 \\
& & & & \\
& & & &
\end{tabular}

(b)

the resulting probability maps were used as prior information for segmentation of the manually segmented subject. The segmentations were then compared with the manual segmentation using Dice metric (Fig. 10). In this experiment the EM segmentation with non-rigidly warped blurred manual segmentation performed best overall whereas the local method was the most successful in the thalamus area.

\section{Discussion and Conclusion}

In this paper we have described the problem segmentation of the brain in early childhood with focus on segmentation of deep grey matter structures. Intensity based methods are likely to fail in this area as the voxel intensities are brighter than in cortical grey matter. This is caused by different composition of deep grey matter and presence of white matter tracts. However it is necessary to preserve the correct structure boundaries when performing white and grey matter classification. We showed that it is possible to substantially improve the results by using correct population-specific prior information. We also showed how to create such population specific probabilistic atlas. In future we would like to focus on development of a robust method which would perform tissue classification locally and incorporate iterative non-rigid warping of deformable atlas into segmentation process.

\section{References}

1. Marlow, N., Wolke, D., Bracewell, M.A., Samara, M., the EPICure Study Group: Neurologic and developmental disability at six years of age after extremely preterm birth. Pediatrics 115(2) (2005) 286-94

2. Wilke, M., Schmithorst, V., Holland, S.: Normative pediatric brain data for spatial normalization and segmentation differs from standard adult data. Magnetic Resonance in Medicine 50(4) (2003) 749-757

3. Leemput, K.V., Maes, F., Vandermeulen, D., Suetens, P.: Automated model-based tissue classification of MR images of the brain. IEEE Transactions on Medical Imaging 18(10) (1999) 897-908 
4. Leemput, K.V., Maes, F., Vandermeulen, D., Suetens, P.: Automated model-based bias field correction of MR images of the brain. IEEE Transactions on Medical Imaging 18(10) (1999) 885-896

5. Tasdizen, T., Awate, S.P., Whitaker, R.T., Foster, N.L.: MRI tissue classification with neighborhood statistics: A nonparametric, entropy-minimizing approach. In: MICCAI '05, Part II. Lecture Notes in Computer Science (2005) 517-525

6. Ashburner, J.: Computational Neuroanatomy. PhD thesis, University College London (2000)

7. Wells III, W.M., Grimson, W.E.L., Kikinis, R., Jolesz, F.A.: Adaptive segmentation of MRI data. IEEE Transactions on Medical Imaging 15(4) (1996) 429-442

8. Joshi, N., Brady, M.: A non-parametric mixture model for partial volume segmentation of MR images. In: British Machine Vision Conference. (2005)

9. Ren, J., Sneller, B., Rueckert, D., Hajnal, J., Hill, D.: A comparison of tissue type labelings of direct classification and segmentation propagation techniques for MR brain images. In: Proc. of Medical Image Understanding and Analysis '05. (2005) $55-58$

10. Zhang, Y., Brady, M., Smith, S.: Segmentation of brain MR images through a hidden markov random field model and the expectation maximization algorithm. IEEE Transactions on Medical Imaging 20(1) (2001) 45-57

11. Rueckert, D., Sonoda, L.I., Hayes, C., Hill, D.L.G., Leach, M.O., Hawkes, D.J.: Non-rigid registration using free-form deformations: Application to breast MR images. IEEE Transactions on Medical Imaging 18(8) (1999) 712-721

12. Pohl, K., Wells, W., Guimond, A., Kasai, K., Shenton, M., Kikinis, R., Grimson, W., Warfield, S.: Incorporating non-rigid registration into expectation maximization algorithm to segment MR images. In: MICCAI '02. Volume 2488 of Lecture Notes in Computer Science. (2002) 564-572

13. D'Agostino, E., Maes, F., Vandermeulen, D., Suetens, P.: Non-rigid atlas-to-image registration by minimization of class-conditional image entropy. In: MICCAI '04, Part I. Lecture Notes in Computer Science (2004) 745-753

14. Pohl, K.: Prior Information for Brain Parcellation. PhD thesis, Massachusetts Institute of Technology (2005)

15. Ashburner, J., Friston, K.: Unified segmentation. NeuroImage 26 (2005) 839-851

16. Warfield, S.K., Kaus, M., Jolesz, F.A., Kikinis, R.: Adaptive template moderated spatially varying statistical classification. In: MICCAI '98. (1998) 431-438

17. Chris A. Cocosco, A.P.Z., Evans, A.C.: A fully automatic and robust brain MRI tissue classification method. In: Medical Image Analysis. Volume 7. (2003) 513-527

18. Prastawa, M., Gilmore, J.H., Lin, W., Gerig, G.: Automatic Segmentation of Neonatal Brain MRI. In: MICCAI '04, Part I. Lecture Notes in Computer Science (2004) 10-17

19. Sled, J.G.; Zijdenbos, A.E.A.: A nonparametric method for automatic correction of intensity nonuniformity in MRI data. IEEE Transactions on Medical Imaging 17(1) (1998) 87-97

20. Statistical Parametric Mapping. (www.fil.ion.ucl.ac.uk/spm)

21. Expectation Maximization Segmentation. (www.medicalimagecomputing.com/ EMS) 